\title{
The Improved Efficiency of Polymer Solar Cells by Fluorine Atoms at Ortho-Position of Alkxoyphenyl Group in Benzodithiophene (BDT) Units
}

\author{
Wenfei Shen ${ }^{1}$, Jianguo Tang ${ }^{1, *}$, Ning Wang ${ }^{2}$, Yao Wang ${ }^{1}$, Jixian Liu, Linjun Huang ${ }^{1}$, Jiuxing Wang ${ }^{1}$, \\ Yanxin Wang ${ }^{1}$, Wei Wang ${ }^{1}$, Renqiang Yang ${ }^{2 *}$, Laurence A. Belfiore ${ }^{1,3, *}$ \\ ${ }^{1}$ Academy of Hybrid Materials, National Base of International Sci. \& Tech. Cooperation on Hybrid \\ Materials, Qingdao University, 308 Ningxia Road, Qingdao 266071, P. R. China \\ ${ }^{2}$ Qingdao Institute of Bioenergy and Bioprocess Technology, Chinese Acadamy of Sciences, 189 \\ Songling Road, Qingdao, 266101, P.R.China \\ ${ }^{3}$ Department of Chemical and Biological Engineering, Colorado State University, Fort Collins, \\ Colorado 80523, USA \\ *E-mail: jianguotangde@ @otmail.com; belfiore@engr.colostate.edu
}

doi: $10.20964 / 2017.07 .07$

Received: 15 March 2017 / Accepted: 30 April 2017 / Published: 12 June 2017

In this article, two novel donor polymers containing fluorine atoms at ortho-position of alkxoyphenyl group in benzodithiophene (BDT) units are constructed. Compared with the reference polymer(P1) without fluorine atom, the polymers with fluorine atoms (P2 and P3) show their great different electrochemical properties, optical properties, morphology and hole mobility. Most significant change is their photovoltaic performances. The devices formed by P2 and P3 show much higher power conversion efficiency (PCE), 5.44\% and $6.12 \%$, respectively. The reasons should be that the incorporation of fluorine atom at ortho-position of alkoxyphenyl side chains increases the oxidation potential, decreases the highest occupied molecular orbit (HOMO) level of donor polymers, and thus increases the open circuit voltage (Voc) in device. Moreover, the linear hexyl side chain of P3 causes much higher PCE because it generates better molecular aggregation and hole transport in device.

Keywords: new donor materials, fluorine atom incorporation, increase oxidation potential, better molecular aggregation, polymer solar cell.

\section{$\underline{\text { FULL TEXT }}$}

(C) 2017 The Authors. Published by ESG (www.electrochemsci.org). This article is an open access article distributed under the terms and conditions of the Creative Commons Attribution license (http://creativecommons.org/licenses/by/4.0/). 